\title{
RECENT PHYSICS RESULTS FROM DELPHI
}

\author{
P.B. Renton
}

To Appear in Modern Physics Letters A 


\title{
RECENT PHYSICS RESULTS FROM DELPHI
}

\author{
P.B. RENTON
}

Particle and Nuclear Physics Laboratory, University of Oxford, Oxford OX1 3RH

\section{Introduction}

The $*$ Standard SU(3) $\times \mathrm{SU}(2) \times \mathrm{U}(1)$ Model $(\mathrm{SM})$ has proven to be remarkably successful. The predictions of the $\mathrm{SU}(2)_{L} \times \mathrm{U}(1)_{Y}$ electroweak sector are compatible with neutral current data from a wide range of processes and energy scales. The $\mathrm{SU}(3)_{\text {colour }}$ sector (Quantum Chromodynamics) has proven to be more difficult to test because of the inherent uncertainties in going from the perturbative calculations involving quarks and gluons, to the observed jet-like structures in hadronic final states. The advent of the Large Electron Positron collider (LEP) at CERN, operating at the $Z^{\circ}$ resonance, has allowed tests to be performed in the electroweak sector to a hitherto unobtainable precision and to measure precisely QCD phenomena in clean, well defined experimental conditions, and at jet energies significantly higher than in previous $e^{+} e^{-}$studies. This paper contains a brief review of results obtained with the DELPHI detector. More details of the analysis and a complete list of references can be found in the papers cited below.

\section{Data Collection}

Since the remarkably successful start-up of LEP in August 1989 the DELPHI detector has collected about 137,000 hadronic $Z^{\circ}$ decays $(83,000$ up to mid-July 1990 , on which the results below are based). A detailed description of the DELPHI detector can be found in ref.[1]. Charged tracks are reconstructed in the $1.2 \mathrm{~T}$ magnetic field, generated by a large superconducting solenoid, using the time projection chamber (TPC), together with the inner and outer detectors (ID and OD). In the end-cap region, forward chambers (FCA and FCB) complete the tracking. Electromagnetic calorimetry is achieved by high density projection chambers (HPC) in the barrel region and by large lead-glass arrays in the forward directions (FEMC). The iron return yoke of the magnet is instrumented with a hadron calorimeter (HCAL) in both the barrel and end-caps and two double layers of drift chambers are positioned towards and on the outside of the iron for muon identification (MUC). DELPHI is triggered by a series of flexible and redundant combinations of signals from the tracking chambers, the electromagnetic calorimeters, the muon chambers and by large scintillator hodoscopes in the barrel and forward regions.

The luminosity is determined by measurement of the small angle Bhabha scattering process $\left(e^{+} e^{-} \rightarrow e^{+} e^{-}\right.$, in the angular range $\left.43 \leq \theta \leq 135 \mathrm{mrad}\right)$ using a small angle tagger (SAT) [2]. This consists of two calorimeters made of lead and scintillating fibres, one of which is equipped with a precisely constructed lead mask to define accurately the inner radius of the acceptance. This is important as the cross section $\alpha \theta^{-3}$. A further mask covers the region where the two half-cylinders are joined. On the opposite side a silicon tracker is installed in front of the calorimeter. The estimated systematic error on the luminosity for the 1990 data is $1.7 \%$, with an additional theoretical uncertainty of $1.0 \%$ arising from lack of knowledge of higher order terms. Further work on the sources of systematic error is expected to reduce the experimental component of this error to $1 \%$ or better. 
In addition to the above components, DELPHI is also equipped with a two-layer silicon strip microvertex detector, which has been successfully commissioned. Tests of the ring image Cerenkov (RICH) counters have also proved successful and these devices will considerably enhance the future physics capabilities of DELPHI, particularly in the area of flavour tagging.

\section{3. $Z^{\circ}$ line-scan}

Measurement of the cross section $e^{+} e^{-} \rightarrow f \bar{f}$ around the $Z^{\circ}$ pole allows a precise determination of the $Z^{\circ}$-fermion neutral current couplings. In the (modified) Born approximation, the cross section, as a function of the centre of mass energy $(\sqrt{s})$, is

$$
\sigma_{f}(s)=\frac{s}{\left(s-M_{z}^{2}\right)^{2}+s^{2} \Gamma_{z}^{2} / M_{z}^{2}}\left(\frac{12 \pi \Gamma_{e} \Gamma_{f}}{M_{z}^{2}}\right)[1+\delta(s)]
$$

where $M_{z}, \Gamma_{z}$ and $\Gamma_{f}$ are the $Z^{\circ}$ mass, total width and partial decay width for $Z^{\circ} \rightarrow f \bar{f}$ respectively and $\delta(s)$ accounts for the effects of radiative corrections. In addition to the above $Z^{\circ}$ term, there is a much smaller photon contribution and a $\gamma-Z^{\circ}$ interference term. The partial width $\Gamma_{f}$ can be written

$$
\Gamma_{f}=\frac{G_{F} M_{z}^{3}}{6 \sqrt{2} \pi}\left(v_{f}^{2}+a_{f}^{2}\right)
$$

where $G_{F}$ is the Fermi constant, and $v_{f}$ and $a_{f}$ are the vector and axial-vector couplings, which are completely specified in the Standard Model. For charged leptons, to lowest order, $v_{\ell}=-1 / 2+2 \sin ^{2} \theta_{W}$ and $a_{\ell}=-1 / 2$.

The forward-backward asymmetry

$$
A_{F B}^{f}(\sqrt{s})=\frac{\sigma_{F}^{f}-\sigma_{B}^{f}}{\sigma_{F}^{f}+\sigma_{B}^{f}}
$$

has also been measured, where $\sigma_{F}^{f}\left(\sigma_{B}^{f}\right)$ is the cross-section for the production of $f$ with $\cos \theta>0$ $(<0)$, with $\theta$ the angle between $f$ and the incident $e^{-}$. The tree level asymmetry for the $Z^{\circ}$ term, at the pole, is

$$
A_{F B}^{f}=\frac{3}{4} A_{e} A_{f}, \quad \text { where } A_{f}=\frac{2 v_{f} a_{f}}{v_{f}^{2}+a_{f}^{2}} .
$$

The above formulae, in particular that for the cross-section line-shape, are considerably modified by higher order radiative (in particular electromagnetic) corrections. These can be computed precisely, and are taken into account in the analysis using the method of Bardin et al. [3].

The selection criteria and correction factors for the final states $e^{+} e^{-} \rightarrow$ hadrons and $e^{+} e^{-} \rightarrow \ell^{+} \ell^{-}(\ell=e, \mu$ and $\tau)$ are similar to those described in refs.[2] and [4]. The hadronic event selection is based on charged tracks measured in the barrel region $\left(n_{c h} \geq 5, \Sigma E_{c h} \geq\right.$ $0.14 \sqrt{s}$ ). The addition of further triggers in 1990 leads to an improved trigger efficiency of $(99.5 \pm 0.2) \%$. The overall detection efficiency is $(92.7 \pm 1.1) \%$, with a contamination from $\tau \tau$ of $(0.3 \pm 0.1) \%$. The initial selection of leptonic events is based on their low multiplicity. For $e^{+} e^{-} \rightarrow e^{+} e^{-}$events the electrons are identified using the HPC. The muons in the $e^{+} e^{-} \rightarrow \mu^{+} \mu^{-}$ final state are identified by either the HPC, HCAL or MUC. The selection of $e^{+} e^{-} \rightarrow \tau^{+} \tau^{-}$ is based on the $1-n(1 \leq n \leq 5)$ topology, excluding $e^{+} e^{-}$and $\mu^{+} \mu^{-}$final states by cuts on acollinearity and acoplanarity. The leptonic events are required to have at least one track in the polar angle range $43^{\circ} \leq \theta \leq 137^{\circ}$. After the requirement that all the relevant sub-detector components were fully functional, the resulting event samples are about 68,000 hadronic decays and 4,000 leptonic decays. The estimated systematic errors on the corrected numbers of leptonic events are $1.6 \%, 1.9 \%$ and $2.7 \%$ for the $e e, \mu \mu$ and $\tau \tau$ final states respectively [5].

\section{Results of fits}

The cross-sections for $e^{+} e^{-} \rightarrow$ hadrons and for $e^{+} e^{-} \rightarrow \ell^{+} \ell^{-}(\ell=e, \mu, \tau)$, as a function of $\sqrt{s}$, for the data taken during 1989 and 1990 are shown in fig.1. Also shown is a 4-parameter 
fit, performed using the program ZFITTER [3]. For the $e^{+} e^{-}$final state, a subtraction of the $t$-channel contribution was made using the program ALIBABA [6]. The fit gives

$$
\begin{aligned}
M_{z} & =91.188 \pm 0.013 \text { (stat) } \pm 0.02 \text { (syst) } \mathrm{GeV}, \\
\Gamma_{z} & =2476 \pm 26 \quad \text { (stat) } \pm 10 \text { (syst) } \mathrm{MeV}, \\
\Gamma_{h} & =1756 \pm 23 \quad \text { (stat) } \pm 20 \text { (syst) } \mathrm{MeV} \quad \chi^{2}=46.8 / 57 \text { d.f. } \\
\text { and } R & =\Gamma_{h} / \Gamma_{\ell}=21.00 \pm 0.38 \text { (stat) } \pm 0.29 \text { (syst). }
\end{aligned}
$$

The systematic error on $M_{z}$ is from the uncertainty in the absolute energy scale of LEP, and is estimated to be about $0.02 \mathrm{GeV}$.

The quantity $R$ is rather insensitive to the (unknown) top-quark mass and is a sensitive test of the validity of the SM. The predicted value is $R=20.69 \pm 0.15$ for $50 \leq m_{t} \leq 250 \mathrm{GeV}$ and $30 \leq m_{H} \leq 1000 \mathrm{GeV}$, and is thus in good agreement with the data. Equivalently, the above fit gives

$$
\begin{aligned}
\Gamma_{\ell} & =83.7 \pm 1.0 \text { (stat) } \pm 1.1 \text { (syst) } \mathrm{MeV} \\
\text { and } \Gamma_{i n v} & =469 \pm 19 \text { (stat) } \pm 22 \text { (syst) } \mathrm{MeV},
\end{aligned}
$$

where $\Gamma_{i n v}=\Gamma_{z}-\Gamma_{h}-3 \Gamma_{\ell}$ is the 'invisible' width. Using the SM value $\Gamma\left(Z^{\circ} \rightarrow \nu \bar{\nu}\right)=166.6$ $\mathrm{MeV}$, the number of light neutrino generations is found to be

$$
N_{v}=2.82 \pm 0.11 \text { (stat) } \pm 0.13 \text { (syst), }
$$

fully compatible with the simplest SM assignment $N_{v}=3$. Lepton universality is assumed in this fit. If this assumption is not imposed the values of $\Gamma_{e}, \Gamma_{\mu}$ and $\Gamma_{\tau}$ are all within one standard deviation of the above value for $\Gamma_{\ell}$. In the SM the leptonic width can be expressed in terms of an effective weak mixing angle, giving

$$
\sin ^{2} \bar{\theta}_{W}=0.2309 \pm 0.0048
$$

A fit to the hadronic cross-section alone gives similar values for $M_{z}$ and $\Gamma_{z}$, and a value for the peak cross-section in the Born approximation, which is also insensitive to $M_{t o p}$, of $\sigma_{0}=42.38 \pm 0.30$ (stat) \pm 0.97 (syst) nb., compared to the SM value of $41.5 \pm 0.1 \mathrm{nb}$.

The forward-backward asymmetries have been determined for $e^{+} e^{-} \rightarrow \mu^{+} \mu^{-}$and $e^{+} e^{-} \rightarrow$ $\tau^{+} \tau^{-}$, and are shown in fig.2, together with the results of a fit which gives

$$
\begin{aligned}
& \mathrm{v}_{\ell}=-0.055_{-0.017}^{+0.024} \text { (stat) } \pm 0.008 \text { (syst) } \\
& a_{\ell}=-0.502 \pm 0.003 \text { (stat) } \pm 0.005 \text { (syst). }
\end{aligned}
$$

The signs of the couplings are imposed from other measurements. These results, together with the SM values for a range of top-quark and Higgs masses, are shown in fig.3.

\section{Partial widths for heavy quarks}

The partial width $\Gamma_{c}$ for $Z^{0} \rightarrow c \bar{c}$ has been extracted by means of an inclusive analysis of charged pions coming from the decay of the charmed meson $D^{*+} \rightarrow D^{\circ} \pi^{+}$and $D^{*-} \rightarrow \bar{D}^{\circ} \pi^{-}$ [7]. The kinematics of this process are such that the transverse momentum $p_{T}$ of the pion with respect to jet axis is small, $<p_{T}>\sim 65 \mathrm{MeV} / \mathrm{c}$, compared to $300 \mathrm{MeV} / \mathrm{c}$ for the usual fragmentation process. From the observed excess of charged tracks at low $p_{T}$ in hadronic $Z^{\circ}$ decays, together with the probability for a charmed quark to fragment into a $D^{*+}$ (taken from a measurement at lower energy), the branching ratio $R_{c}=\Gamma_{c} / \Gamma_{h}$ is determined to be

and thus

$$
R_{c}=0.162 \pm 0.030 \text { (stat) } \pm 0.050 \text { (syst) }
$$

$$
\Gamma_{c}=284 \pm 53 \text { (stat) } \pm 88 \text { (syst) } \mathrm{MeV} \text {, }
$$

compared to the SM values of $0.171 \pm 0.001$ and $295 \pm 5 \mathrm{MeV}$ respectively.

The partial width $\Gamma_{b}$ for $Z^{\circ} \rightarrow b \bar{b}$ has been measured by using a weighting technique based on the boosted sphericity product, which is a shape variable defined for two jet events [8]. 
The $b$-quark hadrons in the two jets are significantly heavier than those formed in other decay channels, and this variable is sensitive to the resulting kinematics. The measured $b \bar{b}$ branching ratio $R_{b}=\Gamma_{b} / \Gamma_{h}$ is

$$
R_{b}=0.209 \pm 0.030 \text { (stat) } \pm 0.031 \text { (syst) }
$$

and

$$
\Gamma_{b}=367 \pm 53 \text { (stat) } \pm 55 \text { (syst) } \mathrm{MeV} \text {, }
$$

compared to the SM values of $0.218 \pm 0.003$ and $376 \pm 4 \mathrm{MeV}$ respectively.

\section{QCD studies}

Two infrared safe methods have been used to measure the QCD running coupling constant $\alpha_{s}$, using the $\overline{M S}$ renormalisation scheme.

i) Jet multiplicity [9]. The method used consists essentially in merging all pairs of charged hadrons until

$$
y_{i j}=\frac{2 E_{i} E_{j}\left(1-\cos \theta_{i j}\right)}{E_{v i s}^{2}}>y_{c}
$$

where $E_{i}$ and $E_{j}$ are the energies, $\theta_{i j}$ is the opening angle and $E_{v i s}$ is the total charged energy in the event. The fractions of 2,3 and 4 jet events found are compared to a perturbative QCD calculation, made to order $\alpha_{s}^{2}$. The QCD calculation is for partons, and the relationship between the hadron jet multiplicities and those for partons was computed using several options of the LUND JETSET generator. Such generators give a good description of the final state jet and multiplicity properties $[10,11]$. The sensitivity of the result for $\alpha_{s}$ to the value of the cut-off $y_{c}$ was carefully studied. This method gives, for $y_{c}>0.05$,

$$
\alpha_{s}\left(M_{z}^{2}\right)=0.114 \pm 0.003 \text { (stat) } \pm 0.004 \text { (syst) } \pm 0.012 \text { (theory) }
$$

ii) Energy-energy correlations [12]. The energy-energy correlation (EEC) is constructed out of pairs of charged particles as follows

$$
E E C(\chi)=\frac{1}{N} \sum_{k=1}^{N} \sum_{i, j} \frac{E_{i} E_{j}}{E_{v i s}^{2}} \delta\left(\theta_{i j}-\chi\right)
$$

where $N$ is the number of events. A quantity which is very sensitive to the underlying parton QCD effects, but rather insensitive to hadronisation effects, is the asymmetry

$$
A E E C(\chi)=E E C(\pi-\chi)-E E C(\chi) .
$$

Fitting this distribution to a theoretical prediction, calculated to $O\left(\alpha_{s}^{2}\right)$, gives

$$
\alpha_{s}\left(M_{z}^{2}\right)=0.106 \pm 0.003 \text { (stat) } \pm 0.003 \text { (syst) }{ }_{-0.000}^{+0.003} \text { (theory). }
$$

The corresponding QCD scale parameter is

$$
\left.\Lambda_{\overline{M S}}=104_{-20}^{+25} \text { (stat) }\right)_{-20}^{+25} \text { (syst) }{ }_{-0}^{+30} \text { (theory) } \mathrm{MeV} .
$$

The two methods are thus compatible. The theoretical uncertainty arises from both the lack of knowledge of higher order QCD effects (e.g. sensitivity to renormalisation scale) and the remnant uncertainties of the influence of hadronisation effects.

QCD is a non-Abelian gauge theory and thus has a triple-gluon vertex arising from the colour charges of the gluons. Feynman diagrams containing this vertex contribute to events with four or more partons in the final state. Experimental evidence for the existence of this vertex has been obtained from a study of four-jet final states. In QCD, the fermionic Casimir operator is $C_{F}=4 / 3$, the number of colours is $N_{c}=3$ and the trace term $T_{R}=n_{f} / 2$, where $n_{f}$ is the 
number of quark flavours. For an Abelian theory $C_{F}=1, N_{c}=0$ and $T_{R}=3 n_{f}$. A fit to variables found to be sensitive to the effect of the triple-gluon vertex gives

$$
\begin{array}{ll}
N_{c} / C_{F}=2.55 \pm 0.55 \pm 0.45 & (2.25 \text { QCD, 0 Abelian }) \\
T_{R} / C_{F}=0.1 \pm 2.4 & (1.88 \text { QCD, 15 Abelian) }
\end{array}
$$

where the systematic error of \pm 0.45 on $N_{c} / C_{F}$ includes the uncertainties from hadronisation effects. The result is incompatible with the Abelian theory and the triple-gluon vertex is needed to describe the data.

Further studies in the field of hadronisation have been made by investigating the phenomena of intermittency [13], which is concerned with the possible existence of very short-range correlations in rapidity. The factorial moments constructed for this study are reproduced by parton shower hadronisation models.

\section{Particle Searches}

In the minimal Standard Model (MSM), there is one physical neutral Higgs scalar boson, the mass of which is unspecified. The relevant production reaction is $e^{+} e^{-} \rightarrow Z^{*} H^{\circ}$, where the off-mass shell $Z^{\circ}$ decays to $\ell^{+} \ell^{-}(\ell=e, \mu, \tau), q \bar{q}$ or $v \bar{v}$ in the usual way. The Higgs decay is mostly to the heaviest available $f \bar{f}$ pair, so the decay is $H^{\circ} \rightarrow b \bar{b}$ over most of the Higgs mass range accessible at LEP. The signature for a Higgs is thus either two isolated leptons, well separated from the decay hadrons from the Higgs (for $\ell^{+} \ell^{-} H^{\circ}$ ), or a significant amount of missing energy and momentum, recoiling against one or two jets from the Higgs decay (for $\left.v \bar{v} H^{\circ}\right)$.

From the data taken in 1989 the mass range $210 \mathrm{MeV} \leq m_{H} \leq 14 \mathrm{GeV}$ was excluded at the $95 \%$ confidence level (c.l.) [14]. With the inclusion of the 1990 data the low mass region (0 to $210 \mathrm{MeV}$ ) has also been excluded. This region corresponds to a relatively long lived Higgs particle which, for the lower part of the mass range, would decay often outside the detector. The high mass limit has also been significantly improved. The limit from the DELPHI experiment is now

$$
m_{H} \geq 34 \mathrm{GeV}, \quad 95 \% \text { c.l. }
$$

In the minimal supersymmetric extension to the Standard Model (MSSM), there are two charged Higgs $\left(H^{ \pm}\right)$and three neutral Higgs (the scalars $h^{\circ}$ and $H^{\circ}$ and the CP-odd pseudoscalar $\left.A^{\circ}\right)$. The model is highly constrained with two free parameters; for example $\tan \beta=v_{2} / v_{1}$, where $v_{2}$ and $v_{1}$ are the vacuum expectation values of the two Higgs doublets, and $m_{h}$ (the mass of the lightest Higgs with $\left.m_{h}<M_{z}|\cos 2 \beta|\right)$. The production mechanisms are $e^{+} e^{-} \rightarrow h^{\circ} Z^{*}$ and $e^{+} e^{-} \rightarrow h^{\circ} A^{\circ}$. The event topologies and the decay modes depend on both $m_{h}$ and $\tan \beta$, and a variety of search techniques are used [15]. The present limits, displayed as contours on a plot of $m_{h}$ against $\tan \beta$, are shown in fig.4. The upper mass limits are now such that $m_{h}>42 \mathrm{GeV}$ for $m_{h} \simeq m_{A}$, at the $95 \%$ c.l.. The main decay modes expected for a heavy charged Higgs are $H^{ \pm} \rightarrow \tau v$ and $c \bar{s}$, with the relative branching ratio depending on $\tan \beta$. The search using the 1989 data [16] has been extended and masses in the range up to $37-43 \mathrm{GeV}$ are now excluded. These limits also apply to charged pseudoscalars in technicolour models.

Other particle searches now lead to results which are close to the LEP 1 kinematic limits, namely for the top-quark [17], a fourth generation $d$-type quark [17], sleptons and charginos [18] and scalar quarks [19]. The results for neutralinos [18] are more model dependent. Heavy stable particles in the range 25 to $45 \mathrm{GeV}$ are also excluded.

\section{Conclusions}

The results from DELPHI, along with those of the other LEP experiments, are in good agreement with the Standard Model, and impose severe constraints on the existence of phenomena beyond this model.

The value found for $\alpha_{s}$ is consistent with the expected QCD 'running' of this coupling with energy scale from lower energy measurements. Further progress in QCD studies will require improved theoretical calculations. 


\section{Acknowledgements}

I would like to thank my colleagues on the DELPHI experiment, whose diligent work produced the results described in this review.

\section{References}

[1] DELPHI Collaboration, P. Aarnio et al., CERN-PPE/90-128 (1990).

[2] DELPHI Collaboration, P. Abreu et al., Phys. Lett. B241 (1990), 435.

[3] D. Yu Bardin et al., Z. Phys. C44 (1989) 493 and DELPHI note 89-71 Phys 50.

[4] DELPHI Collaboration, P. Abreu et al., Phys. Lett. B241 (1990), 425.

[5] DELPHI Collaboration, P. Abreu et al., CERN-PPE/90-119.

[6] W. Beenakker et al., Univ. of Leiden preprint (1990).

[7] DELPHI Collaboration, P. Abreu et al., CERN-PPE/90-123 (1990).

[8] DELPHI Collaboration, P. Abreu et al., CERN-PPE/90-118 (1990).

[9] DELPHI Collaboration, P. Abreu et al., CERN-PPE/90-89 (1990).

[10] DELPHI Collaboration, P. Aarnio et al., Phys. Lett. B240 (1990), 271.

[11] DELPHI Collaboration, P. Abreu et al., CERN-PPE/90-117 (1990).

[12] DELPHI Collaboration, P. Abreu et al., CERN-PPE/90-122 (1990).

[13] DELPHI Collaboration, P. Abreu et al., CERN-EP/90-78 (1990).

[14] DEL̈PHI Collaboration, P. Abreu et al., Nucl. Phys. B342 (1990), 1.

[15] DELPHI Collaboration, P. Abreu et al., Phys. Lett. 245B (1990), 276.

[16] DELPHI Collaboration, P. Abreu et al., Phys. Lett. 241B (1990), 449.

[17] DELPHI Collaboration, P. Abreu et al., Phys. Lett. 242B (1990), 536.

[18] DELPHI Collaboration, P. Abreu et al., CERN-EP/90-80.

[19] DELPHI Collaboration, P. Abreu et al., CERN-EP/90-79.

\section{Figure Captions}

Fig.1. Cross-sections for $e^{+} e^{-} \rightarrow$ hadrons, $e^{+} e^{-} \rightarrow e^{+} e^{-}, e^{+} e^{-} \rightarrow \mu^{+} \mu^{-}$and $e^{+} e^{-} \rightarrow \tau^{+} \tau^{-}$, as a function of $\sqrt{s}$. The cross sections are corrected to $4 \pi$ angular acceptance. The results of the fit described in the text are also shown.

Fig.2. Forward-backward asymmetry for $e^{+} e^{-} \rightarrow \mu^{+} \mu^{-}$and $e^{+} e^{-} \rightarrow \tau^{+} \tau^{-}$, as a function of $\sqrt{s}$, together with the results of the fit described in the text.

Fig.3. Measure values of $a_{e}$ and $v_{e}$ with the 1 and 2 standard deviation contours. Also shown are the values expected from the Standard Model for $30 \leq m_{H} \leq 1000 \mathrm{GeV}$ and $M_{\text {top }}$ as shown.

Fig.4. Regions in $\tan \beta v m_{h}$ plane excluded in the MSSM Higgs search. The dashed line shows the LEP 1 kinematic limit. 

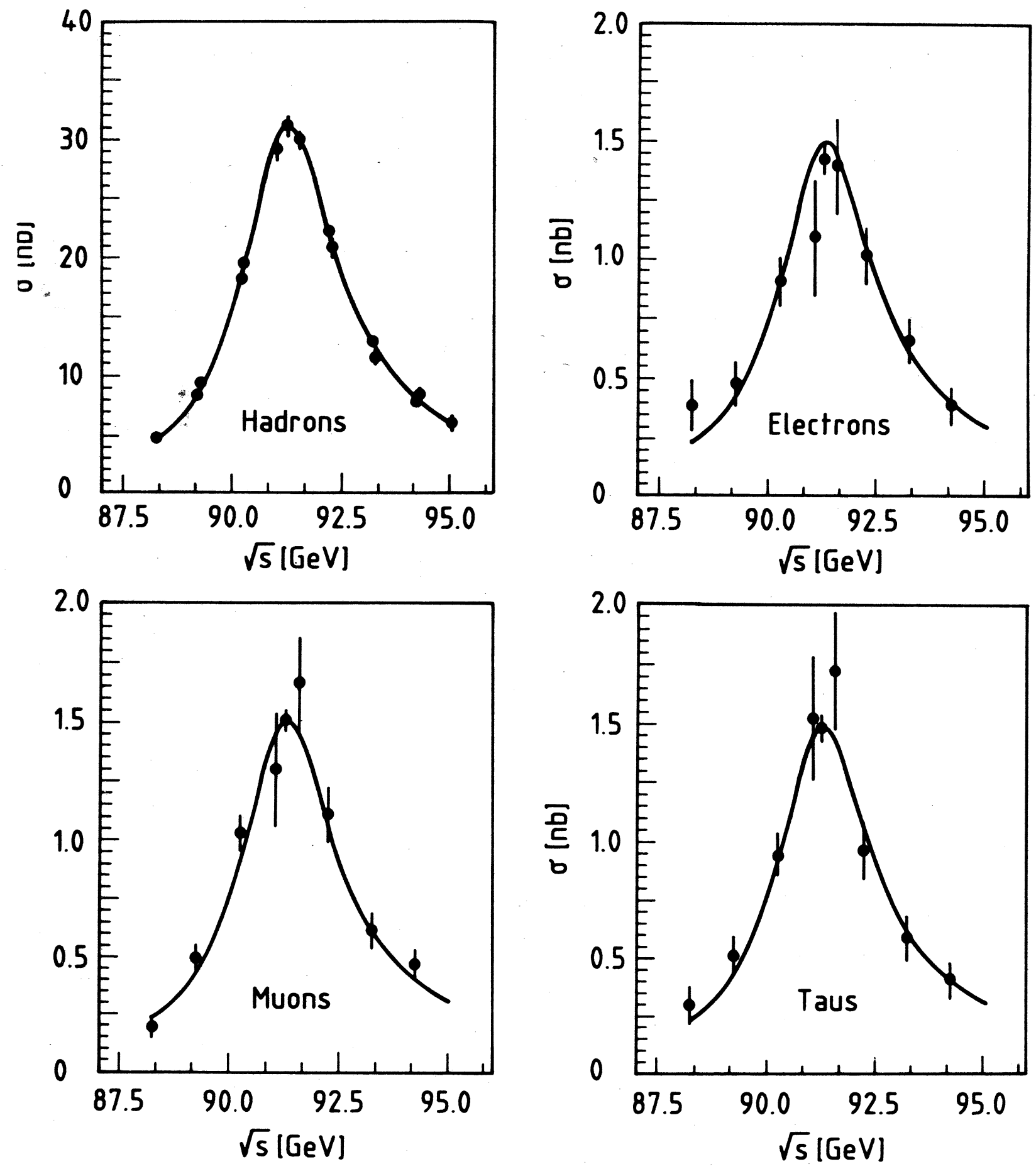

Fig. 1 

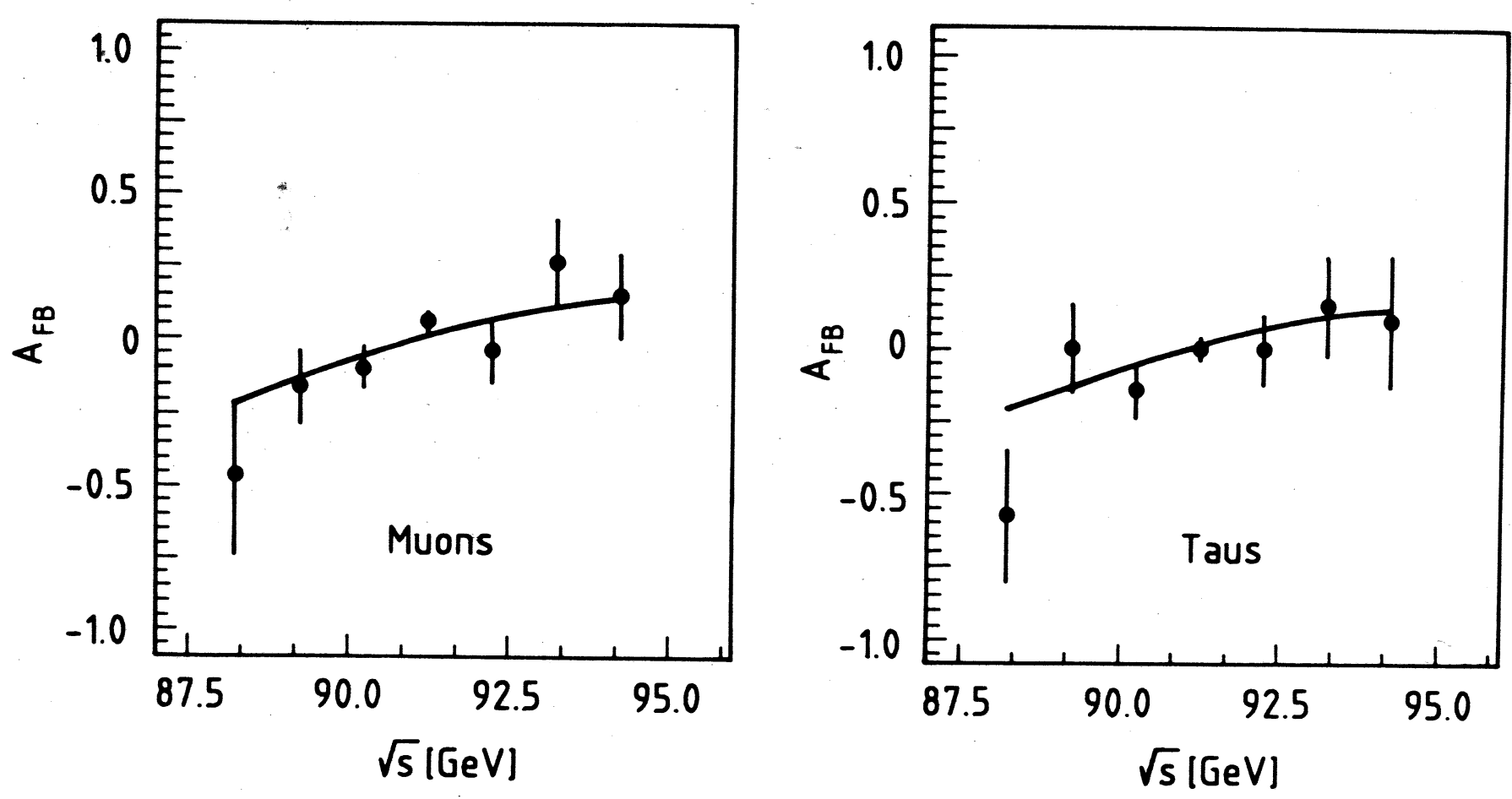

Fig. 2 


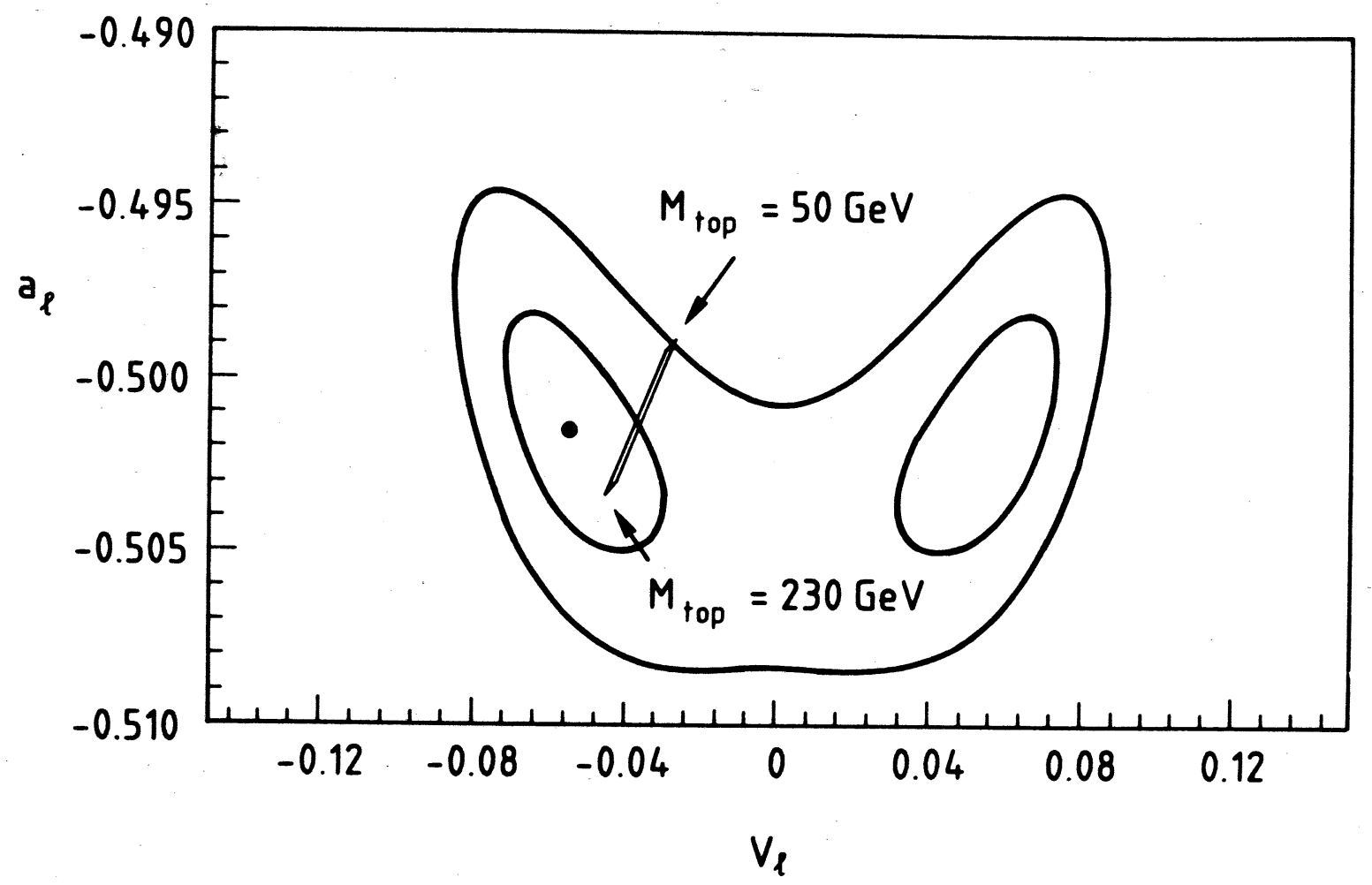

Fig. 3 


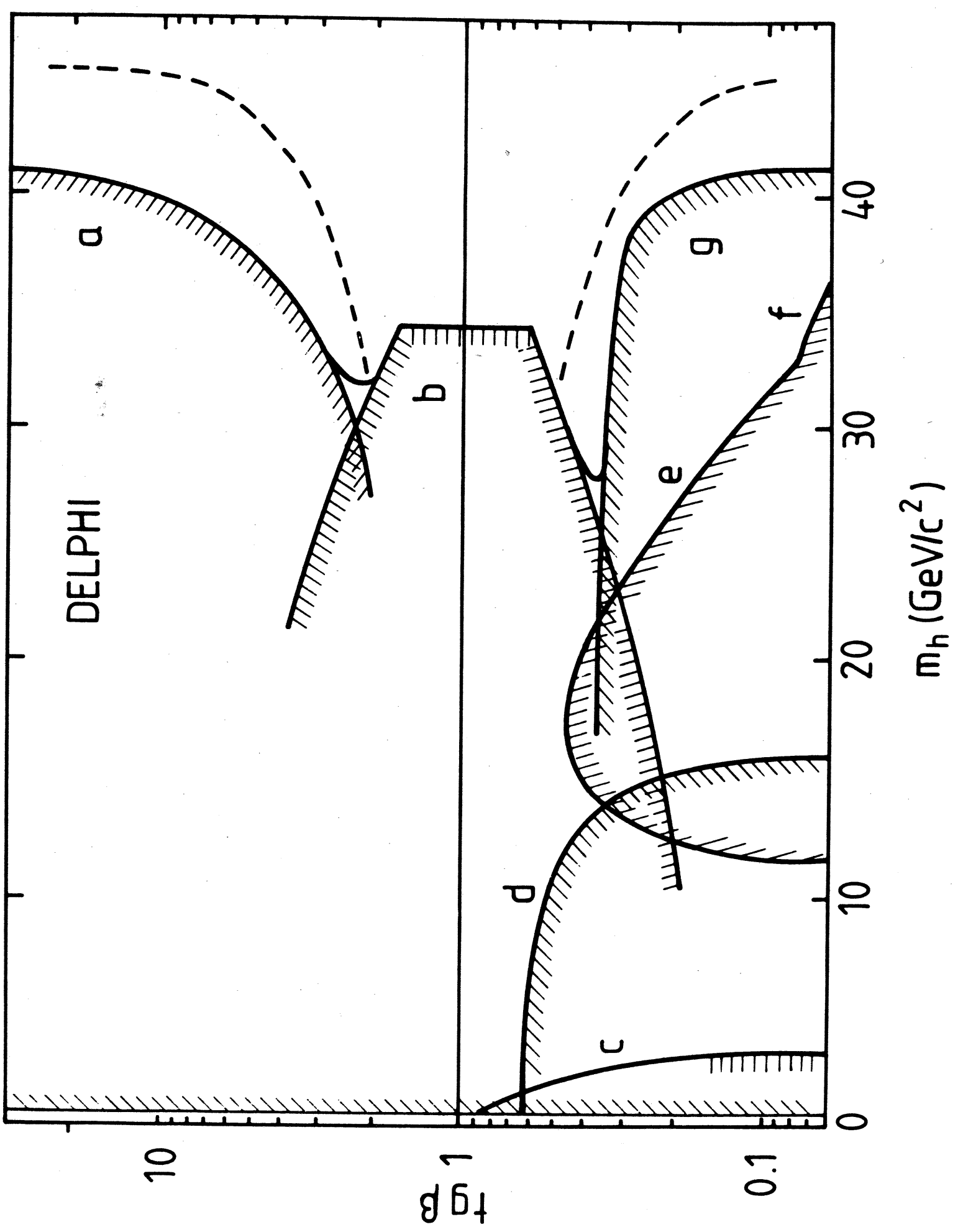

Fig. 4

Imprimé au CERN. Prix : $\quad 0.60 \mathrm{Fr}$. 

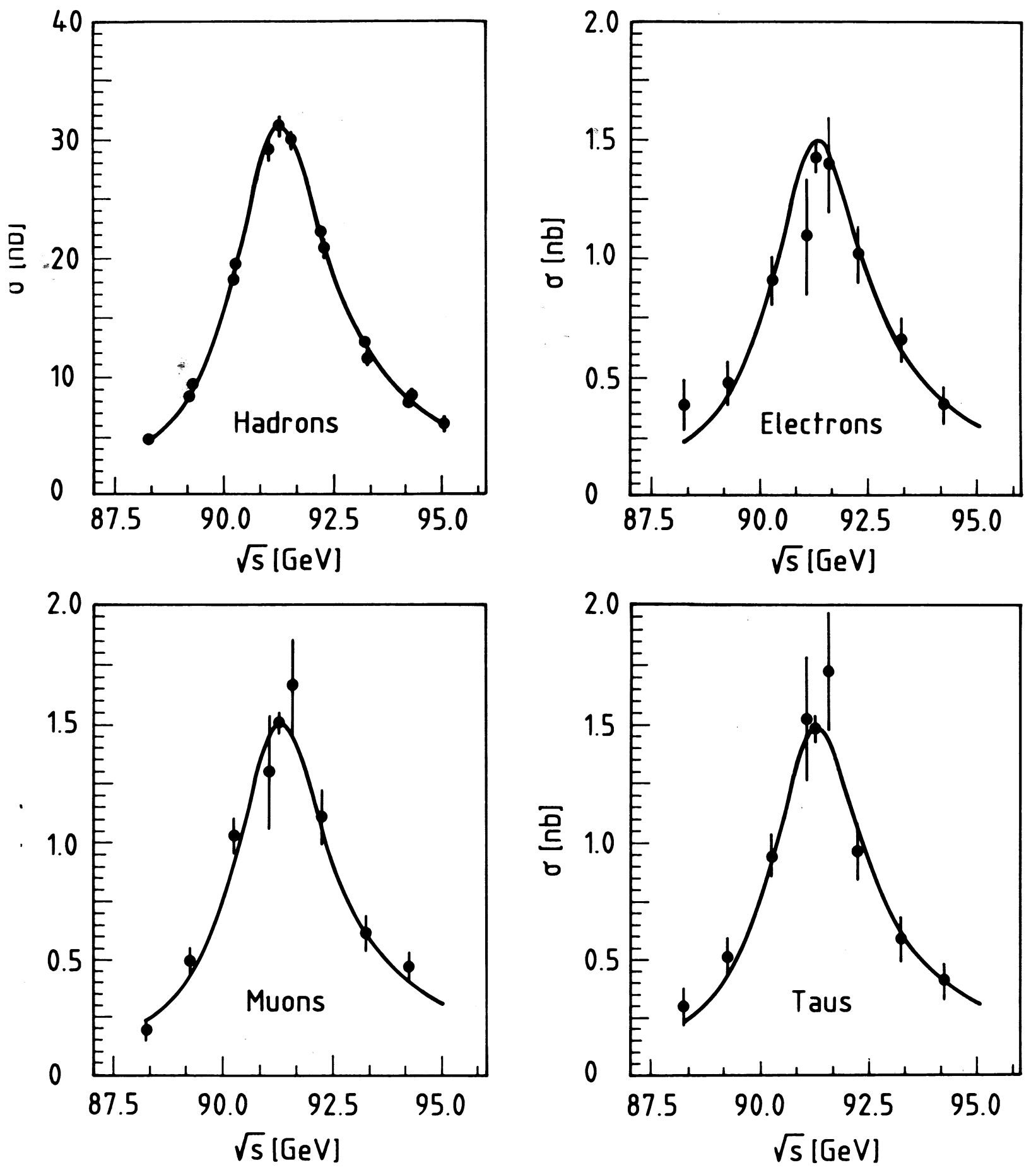

Fig. 1 

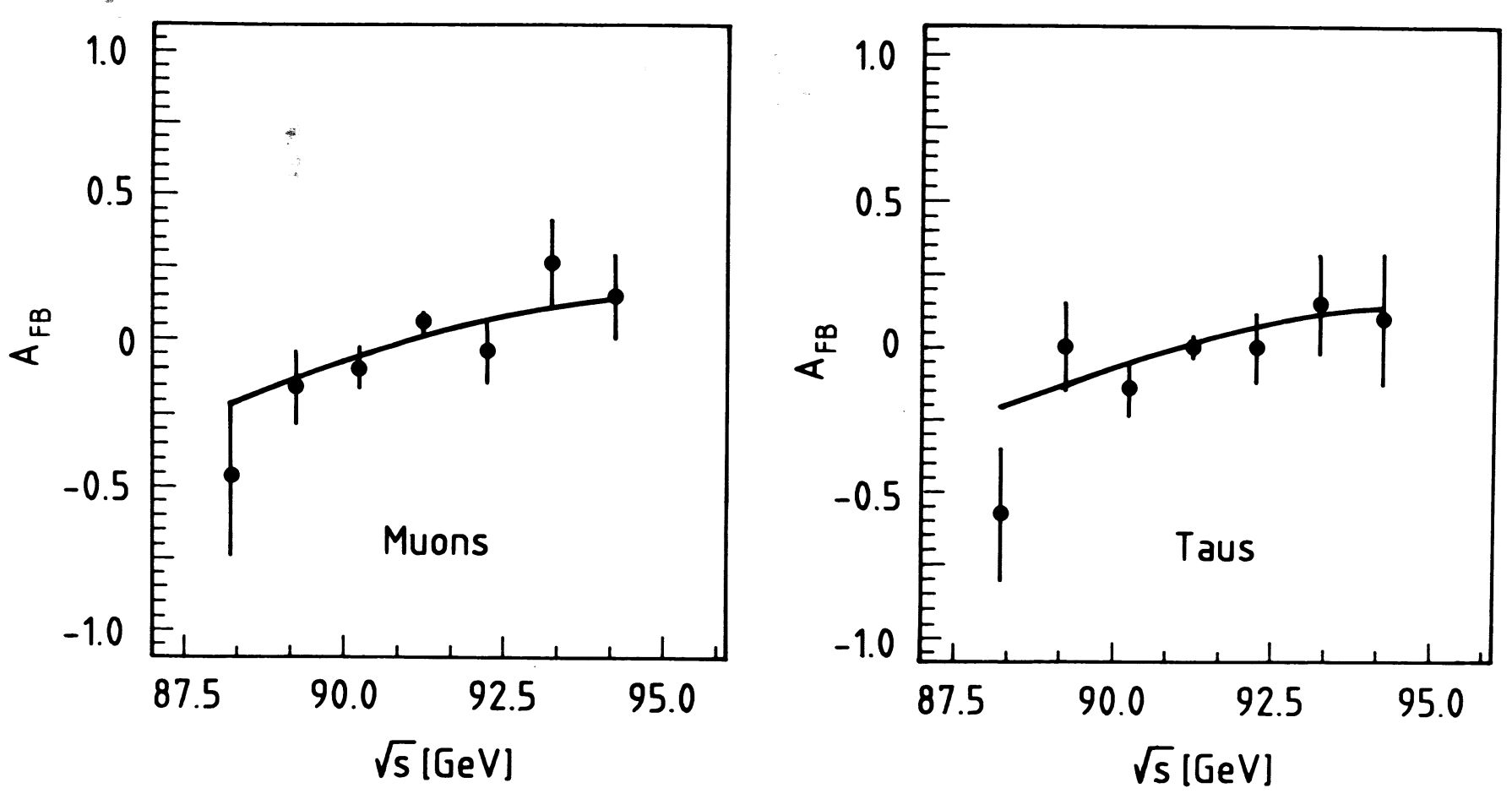

Fig. 2 


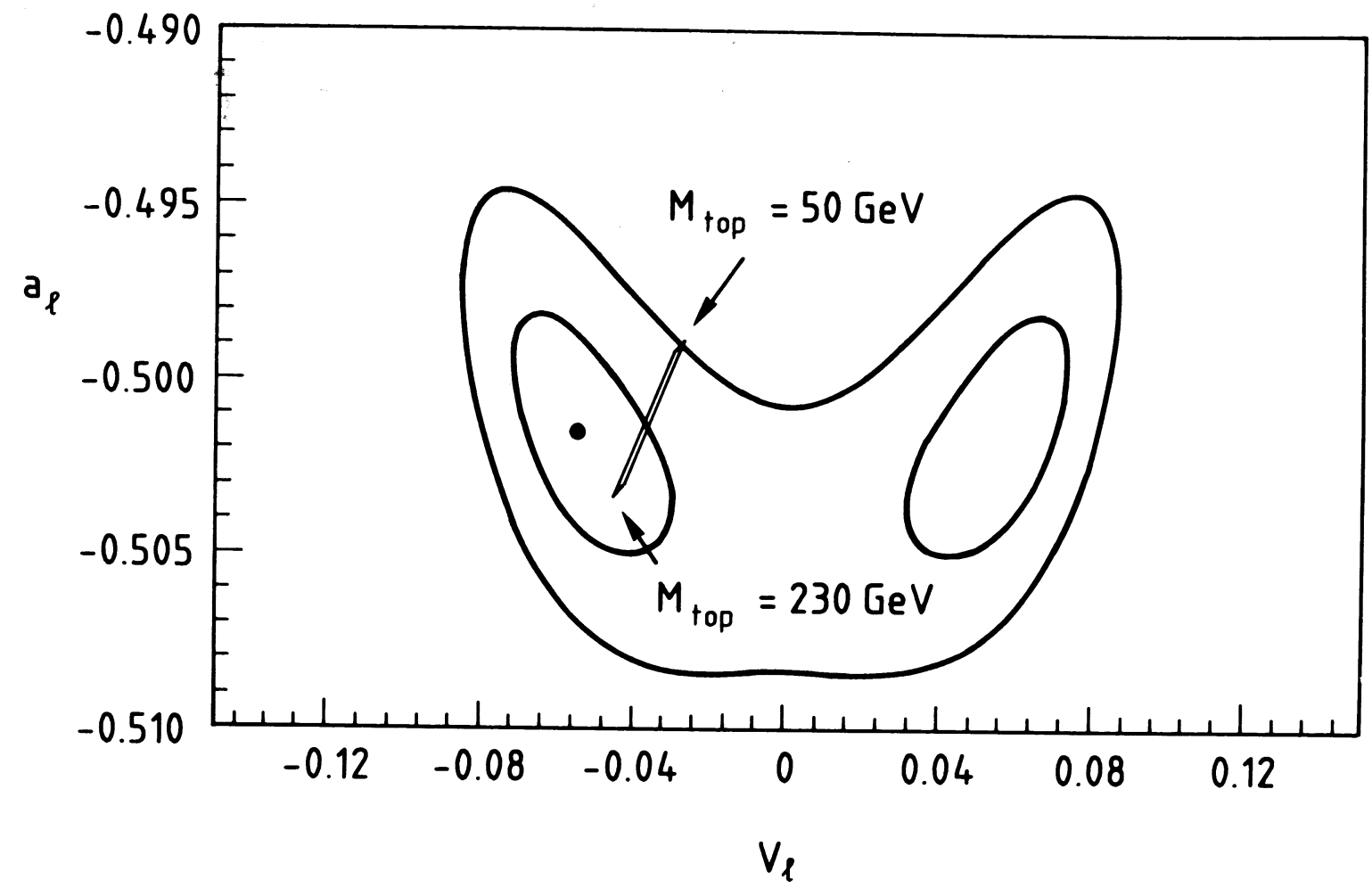

Fig. 3 


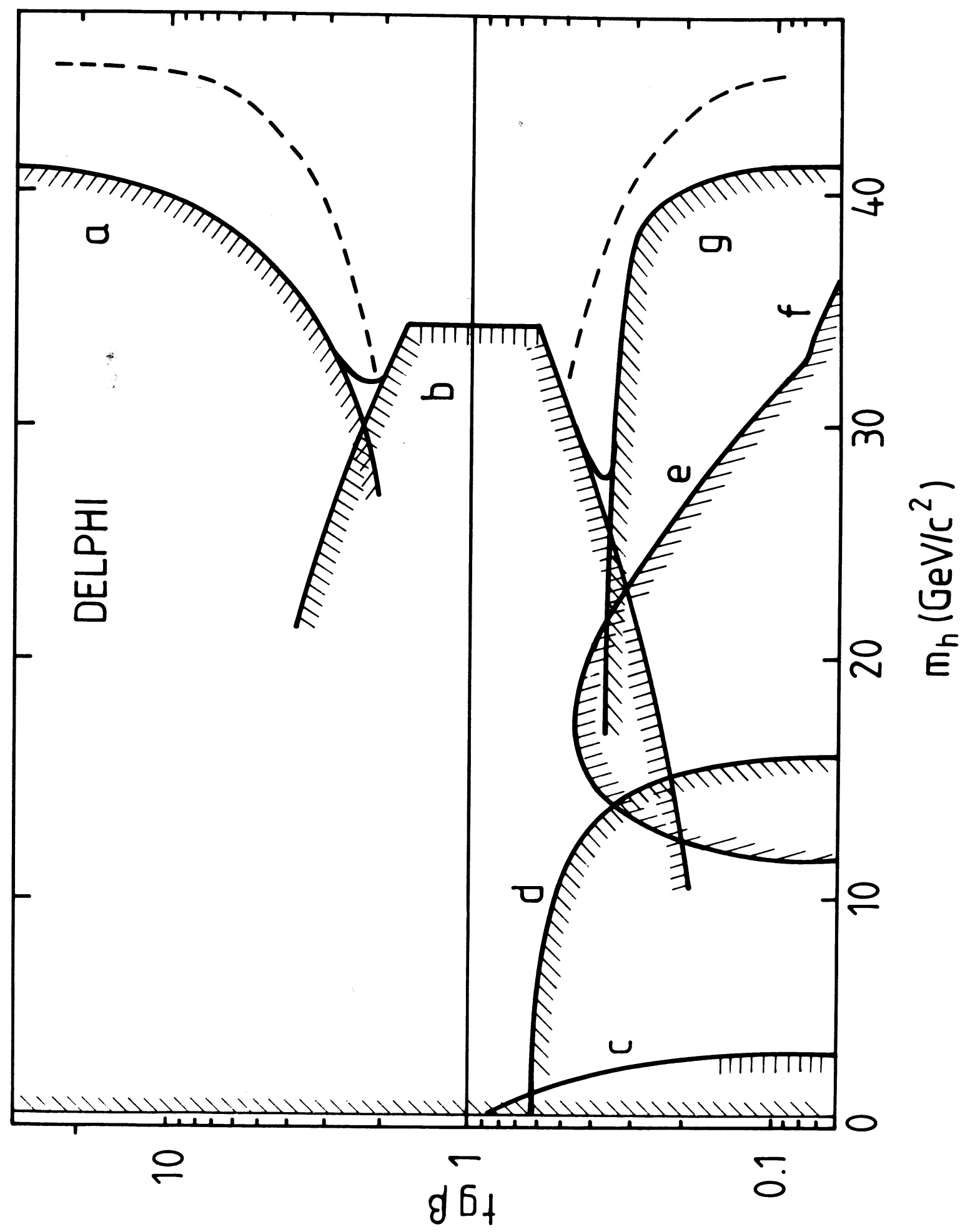

Fig. 4

Imprimé au CERN. Prix : $0.60 \mathrm{Fr}$. 